\title{
Electrically Driven Nonreciprocity Induced by Interband Photonic Transition on a Silicon Chip
}

\author{
Hugo Lira, ${ }^{1}$ Zongfu Yu, ${ }^{2}$ Shanhui Fan, ${ }^{2}$ and Michal Lipson ${ }^{3, *}$ \\ ${ }^{1}$ School of Electrical and Computer Engineering, Cornell University, Ithaca, New York 14853, USA \\ ${ }^{2}$ Department of Electrical Engineering, Stanford University, Stanford, California 94305, USA \\ ${ }^{3}$ Kavli Institute at Cornell for Nanoscale Science, Cornell University, Ithaca, New York 14853, USA
}

(Received 27 March 2012; published 16 July 2012)

\begin{abstract}
We demonstrate electrically driven nonreciprocity on a silicon chip. By achieving an indirect interband photonic transition, we show that the transmission coefficients between two single-mode waveguides become dependent on the propagation directions only in the presence of the electrical drive. Our structure is characterized by a nonsymmetric scattering matrix identical to a linear magneto-optical device.
\end{abstract}

PACS numbers: $42.82 .-\mathrm{m}$

The vast majority of photonic structures are reciprocal. Breaking reciprocity or time-reversal symmetry, as is typically accomplished with magneto-optical effects driven by external or internal magnetic fields, results in new physics such as the emergence of topologically protected one-way photonic edge modes [1-3], as well as a variety of important devices including circulators and isolators, all of which are described by a nonsymmetric scattering matrix [4]. Unfortunately, the magneto-optical effect is not present in standard optoelectronic materials including most metals and semiconductors. While nonmagnetic approaches for achieving optical nonreciprocity have been extensively studied, the experimentally demonstrated approaches thus far however are either nonlinear or introduce additional frequency components. These approaches therefore have not completely reproduced a magneto-optical effect, which is linear and where the input and output waves have the same frequency. In this Letter, we demonstrate an electrically driven nonreciprocity on a silicon chip. By achieving an indirect interband photonic transition $[5,6]$, we show that the transmission coefficients between two single-mode waveguides become dependent on the propagation directions only in the presence of the electrical drive. Importantly, our structure is characterized by a nonsymmetric scattering matrix identical to a linear magnetooptical device.

There have been many previous efforts [6-14] aiming to demonstrate optical nonreciprocity without using magneto-optical effects. These efforts complement the substantial recent progresses [15-19] in miniaturization and integration of magneto-optical devices, by greatly broadening the choice of materials that can be used for demonstrating nonreciprocal physics. By the Lorentz reciprocity theorem, any linear system described by symmetric and time-independent permittivity and permeability tensors is necessarily reciprocal [20]. Therefore, to achieve nonreciprocity without magneto-optics, one necessarily has to rely upon either nonlinear or time-dependent effects. Nonreciprocity based upon $\chi^{(2)}, \chi^{(3)}$, and parametric nonlinearity have been previously demonstrated
$[7,12,13]$. In these nonlinear devices, however, nonreciprocity is power dependent. Thus, their behaviors cannot be characterized by a linear scattering matrix and are fundamentally different from magneto-optical devices. Alternatively, linear nonreciprocity that is independent of signal intensity has been experimentally observed in dynamic structures undergoing electrical modulation $[9,11]$. However, in all previously demonstrated electrically driven structures, the existence of nonreciprocity is associated with significant modulation frequency sidebands $[9,11]$, which again distinguish these devices from magnetooptical effects. While in principle acousto-optic modulation can result in nonreciprocity, the low acousto-optic modulation frequency in the $\mathrm{kHz}$ to $\mathrm{MHz}$ frequency range results in a weak nonreciprocity that can be detected only with very long propagation distances [21].

In this Letter we present an experimental observation of nonreciprocity using interband photonic transition in a silicon waveguide. Photonic transitions in highly confined optical structures, where modes can be carefully manipulated and tailored, are of fundamental interest due to conceptual analogy with electronic transitions in semiconductors [5]. An indirect interband photonic transition occurs between two optical modes having different longitudinal wave vectors (hence the word "indirect"), and having transverse modal profiles with different symmetries (hence the word "interband" since the two modes belong to different photonic bands). Most photonic transitions, including the recent observation of photonic transition in microring resonators [22], and photonic transitions in conventional traveling-wave electroabsorption modulators, are intraband transitions between two modes with the same transverse modal profiles. In intraband transition, the modulation that provides phasematched coupling between two modes at $(\omega, k)$ and $(\omega+\Delta \omega, k+\Delta k)$ will necessarily provide phasedmatched coupling between the modes at $(\omega+\Delta \omega, k+$ $\Delta k)$ and $(\omega+2 \Delta \omega, k+2 \Delta k)$. Thus there is always a cascade of transitions with associated frequency sidebands. As a result, the demonstrated modulation-based isolation 
schemes always have significant frequency components in their output that are different from the input wave frequency. For demonstrating nonreciprocity, interband transition is more attractive since nonreciprocity occurs without the associated frequency sidebands in the intraband transition $[6,13]$.

Demonstrating indirect interband transition is however more challenging. Interband transitions between two different polarizations [21] as can be achieved in acousto-optic modulators, have extremely small operation bandwidth due to the lack of group velocity matching between the two polarizations. In a waveguide system, to induce an interband transition between two modes with different symmetry in its transverse modal profile, the modulation itself cannot be uniform across the waveguide cross section. In addition, since the typical frequency of high speed modulators [22] is on the order of few $\mathrm{GHz}$, the wave-vector difference, between two different transverse modes having frequencies separated by a few $\mathrm{GHz}$, is typically quite large and hence the modulation needs to be specifically constructed. Conventional traveling-wave electro-optic modulator designs cannot generate such a large required modulation wave vector.

Here we demonstrate indirect interband photonic transitions in a slotted waveguide shown in Fig. 1(a). The waveguide supports an even and an odd optical mode [Fig. 1(a)]. The dispersion relation of the waveguide, as shown in Fig. 1(b), is tailored so that the two modes are separated in frequency by only a few $\mathrm{GHz}$, for a difference in wave vector $k=2 \pi / \lambda$, where $\lambda$ is on the order of a few hundreds of microns. This enables one to create an interband transition between the two modes by applying an electrical signal with a $\mathrm{GHz}$ frequency that is achievable in silicon electro-optic devices [23], and to use a structure that is compact (on the order of a few hundred microns in length). In order to induce photonic transition, the overlap integral between the modulation spatial distribution and the initial and final modes should be nonzero [6] and therefore the modulation is applied only to half of the slotted waveguide. Nonreciprocity in such structure is achieved, since the photonic transition does not occur when light propagates in the forward direction from left to right, and occurs only when light propagates in the opposite backward direction [Fig. 1(b)]. For this structure, assuming dimensions $w=450 \mathrm{~nm}, \quad h=$ $215 \mathrm{~nm}, t=35 \mathrm{~nm}$, and $d=500 \mathrm{~nm}$, Fig. 1(c) shows the mode amplitudes in both directions using coupled mode equations [6], for a continuous wave input when the structure is subject to a $10 \mathrm{GHz}$ index modulation. One sees total conversion in the backward direction and minimal conversion $(<2 \%$ in the example shown) in the forward direction. We emphasize that both the modulation and the optical input are continuous wave. The observed nonreciprocity is completely independent of the relative timing between the optical signal and the modulation.
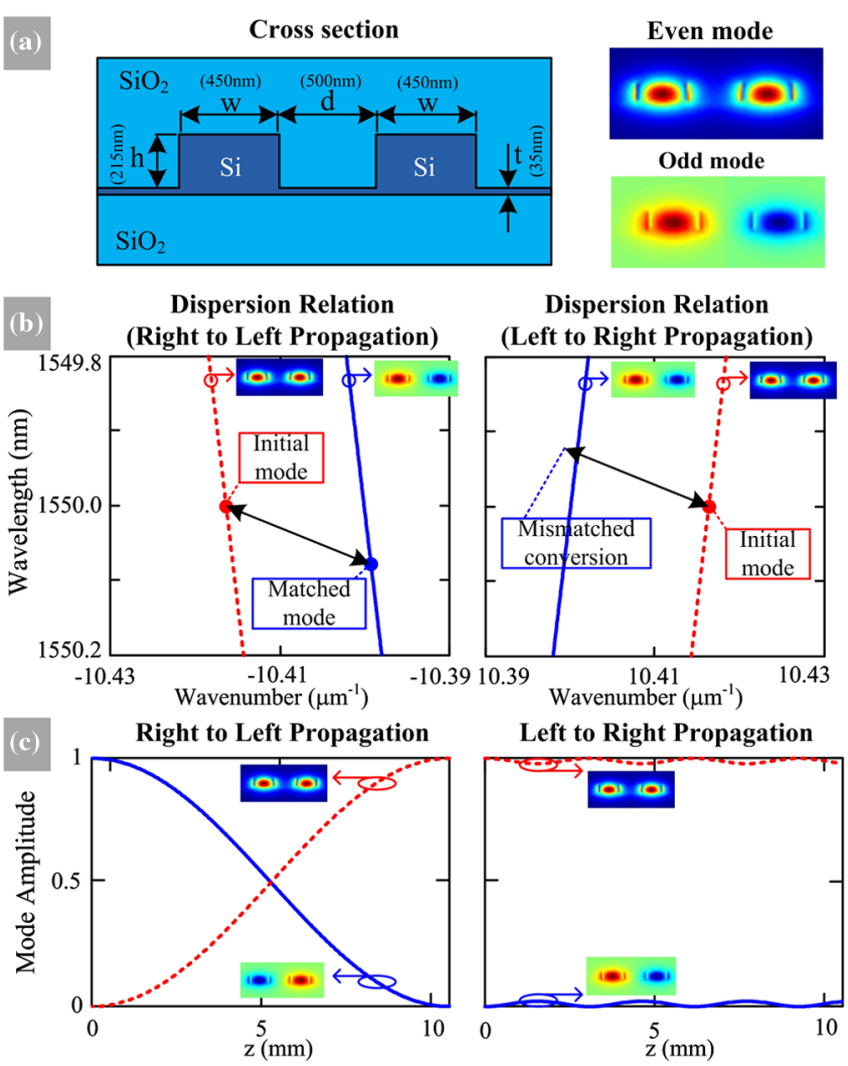

FIG. 1 (color online). (a) Waveguide geometry and materials. (b) Dispersion relation for even (dashed lines) and odd (solid lines) modes of coupled waveguides. The double-ended arrows represent the traveling-wave index modulation. In the left, it matches the initial mode to another mode, while in the right it is observed conversion mismatch. (c) Dynamics of the mode conversion. Right to left propagation achieves full conversion from one mode to the other, while left to right propagation does not.

The most unambiguous demonstration of nonreciprocity is to observe an asymmetry in the transmission coefficients between two single-mode waveguides for the forward and backward transmission directions. We therefore place a $1 \times 2$ MMI (multimode interference waveguide) on both ends of the slotted waveguide [Fig. 2(a)]. The MMI serves to couple between a single-mode waveguide (with an even mode) and the even mode of the slotted waveguide, and to filter away the converted odd mode.

We contrast the two cases where we inject light into the single-mode waveguides along the two directions with the slotted waveguide undergoing modulation that induces interband transition. Figure 2(b) shows a two-dimensional time-domain FEM (finite element method) simulation of the structure demonstrating such a contrast. In the forward direction (left to right), the photonic transition is not allowed, and the mode remains even in the slotted waveguide [Fig. 2(b), top panel]. Thus the structure has a high transmission coefficient. In contrast, in the backward direction (right to left), the transition is allowed and therefore the 


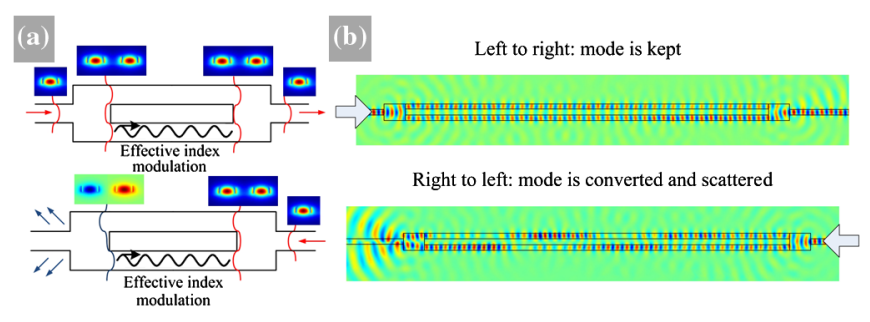

FIG. 2 (color online). (a) Schematic of the isolator. A singlemode waveguide feeds a $1 \times 2 \mathrm{MMI}$, which provides the even mode for the isolator. By modulating the refractive index of one waveguide, we obtain a nonzero overlap between the modes and modulation. In one direction, the even mode is converted to the odd mode, but it is not converted in the other direction. (b) FEM time-domain simulation showing the conversion occurring in only one propagation direction.

even mode injected into the slotted waveguide is completely converted to the odd mode, which is filtered away by the MMI [Fig. 2(b), bottom panel]. As a result, the structure has a low transmission coefficient in the backward direction.

We note that in Fig. 2(b) the converted light, which is at a frequency that is slightly different from the input, is completely radiated away by the MMI. Since the conversion occurs along only one direction, the use of MMI results in a direction-dependent loss that is nonreciprocal. The waves in the two single-mode waveguides only have a single frequency component that is the input frequency. As a result, as far as the wave in the two single-mode waveguides is concerned, the structure is completely characterized by a single $2 \times 2$ scattering matrix with directional-dependent transmission coefficients. From the scattering matrix perspective, the structure is therefore indistinguishable from a magneto-optical device. None of the previously demonstrated nonreciprocal structures that do not use magneto-optical effects have this property.

Based on the theory and numerical simulations above, we experimentally demonstrate the nonreciprocity in interband photonic transition in this system. We place $p n$-junction electrical diodes inside a slotted waveguide, and realize electrical traveling-wave modulation by applying voltages to these diodes through two microwave transmission lines. We use a modulation frequency of $10 \mathrm{GHz}$, which corresponds to the difference in frequency between the two optical modes for the waveguide shown in Fig. 1(b). At this modulation frequency, the electrical wave along the transmission lines has a large period above a centimeter and a corresponding small wave vector, which is not suitable for achieving the interband transition that we require here.

To achieve a larger modulation wave vector, in our structure, the modulation wave vector is instead encoded in the $p n$-junction configurations. We use an array of two different junction regions ( $p n-n p$ and $n p-p n)$ placed in alternating positions along the waveguide, and sinusoidally vary the voltages in the two transmission lines with a $\pi / 2$ difference, as shown in Figs. 3(a) and 3(b). Under an applied bias, only the reverse-biased diodes experience carrier depletion and hence generate significant index change, while the forward-biased diodes experience only minimal carrier leakage and generate no significant index change. In this way, we therefore create a traveling-wave refractive index modulation profile, with a spatial period corresponding to four junction regions along the waveguide [Fig. 3(c)]. Our configuration of junction regions also ensures that index modulation is induced in part of the cross section of the waveguide, fulfilling the required condition of nonzero overlap between the modulation and the product of the profiles of the modes involved.

The achieved modulation of the refractive index along the waveguide is shown in Fig. 3(c). Each circle, square, triangle, and diamond correspond to a diode along the propagation axis. The lines with circles and squares correspond to $p n-n p$ and $n p$ - $p n$ diodes connected to one transmission line, while the lines with diamonds and triangles correspond to the $p n-n p$ and $n p$ - $p n$ diodes connected the second transmission line having a $\pi / 2$ phase difference. The achieved fundamental harmonic of the index modulation is shown with the solid line. One can clearly see that each modulation period $\lambda_{m}$ of $450 \mu \mathrm{m}$ is discretized in

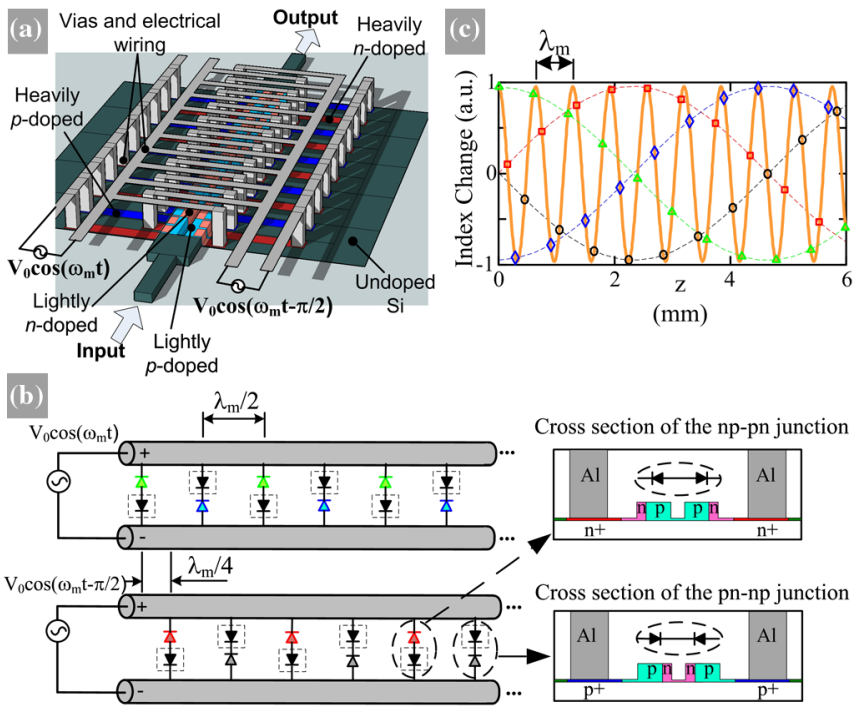

FIG. 3 (color online). (a) Simplified schematics of the device. Undoped, lightly $p$-doped, lightly $n$-doped, heavily $p$-doped, and heavily $n$-doped silicon, as well as vias and electrical wirings are identified. (b) Schematics of the two transmission lines feeding $p n-n p$ and $n p-p n$ junctions. Forward biased diodes have a black dashed square around them, and the other diodes are the reverse biased diodes. The insets show the distribution of dopants across the waveguide forming $n p-p n$ and $p n-n p$ junctions. (c) Normalized index change. Each symbol represents a diode in reverse bias along the waveguide. The lines with triangles and diamonds represent the $p n-n p$ and $n p-p n$ diodes fed by one transmission line, while the lines with squares and circles represent the $p n-n p$ and $n p$ - $p n$ diodes fed by the delayed transmission line. The solid line is the achieved fundamental harmonic of the discretized modulation. 
four parts, with an index change period slightly smaller than $450 \mu \mathrm{m}$ due to the traveling-wave voltage distribution across the transmission line [lines with triangles, diamonds, squares, and circles in Fig. 3(c)].

In our experimental structure, each diode is designed to be $110-\mu \mathrm{m}$ long separated by a $2.5-\mu \mathrm{m}$ region with the opposite dopant providing electrical insulation. Such choice of parameters ensures that the phase matching condition is satisfied at the operation frequency. The overall number of discrete modulation periods is 22 , i.e., 88 modulation sections, or 166 diodes. In order to prevent the periodically loaded transmission lines from having a cutoff below the desired modulation frequency, we add spiral inductors in parallel with each of the $p n-n p$ and $n p-p n$ junctions in the waveguide (with a total length $1.5 \mathrm{~mm}$ corresponding to an $L \approx 1.84 \mathrm{nH}$ ). The length of the stub connecting the capacitors and inductors to the transmission line also affects the cutoff frequency, and from design we expect that a $100-\mu \mathrm{m}$ long stub would push the cutoff above $10 \mathrm{GHz}$. In order to induce maximum index modulation with minimal loss, the dopant concentrations in the $n$-doped and $p$-doped regions were chosen to be $1 \times 10^{18} \mathrm{~cm}^{-3}$ and $1 \times 10^{17} \mathrm{~cm}^{-3}$ respectively, with the center of the doped region shifted about $190 \mathrm{~nm}$ from the center of each of the waveguides, so that losses are minimized for the index change required of the device.

We measure the forward and backward transmission spectra by inputting a continuous wave optical signal, applying a $10 \mathrm{GHz}$ modulation on the structure, and swapping the input and output fibers. Note that the amplitude of the output signal is not affected by the modulation since only the phase (i.e., refractive index) is modulated. Figure 4 shows the ratio between the two spectra. When no electrical signal is applied, the transmission is completely reciprocal with a unity contrast ratio between the forward and backward direction. (The noise measured in the experiments is caused by the mechanical instability of the coupling fiber and can be future reduced.) When the electrical signal is applied, a clear dip appears in the spectrum, indicating nonreciprocity induced by photonic transition, as shown in Fig. 4. As we continue to increase the power of the applied modulation (i.e., stronger coupling between the even and odd modes), we obtain greater contrast between forward and backward transmission. We measured up to $3 \mathrm{~dB}$ contrast when operating at a wavelength of $1558 \mathrm{~nm}$. This contrast is smaller than the one obtained from simulation due to limitations with our electrical signal power supply, which can achieve a maximum $25 \mathrm{dBm}$ output power (or 5.6 $V_{p}$ applied, which might be smaller due to reflections caused by impedance mismatch). For comparison, we show in the bottom right of Fig. 4 the simulated relative transmission using the mode conversion equations and considering the dispersion of the waveguide to determine the bandwidth. One can see that the results agree well with the expected performance of the device.
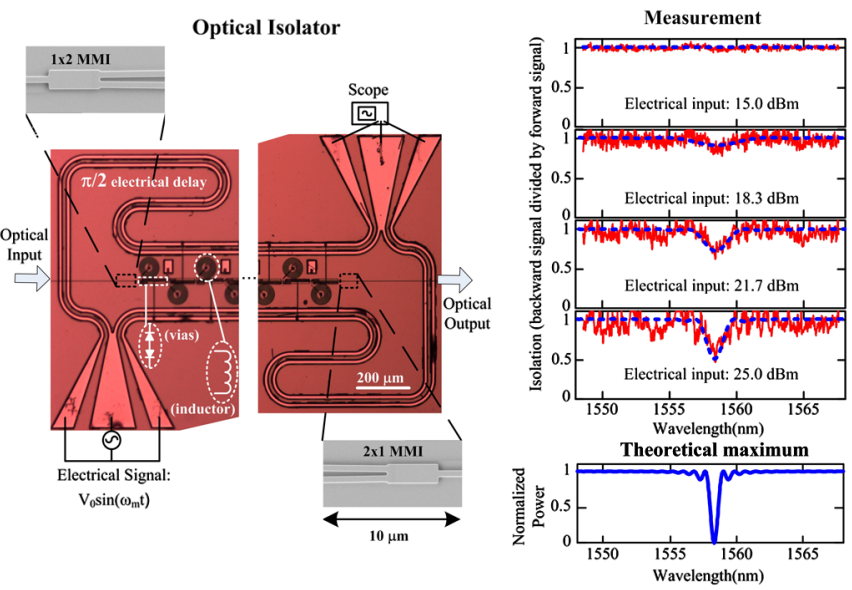

FIG. 4 (color online). In the left we show the electrical elements (optical microscope) and optical elements (scanning electron microscopy) of the electrically driven optical isolator. In the right we show sequentially the increase of isolation measured as a function of electrical signal input power (solid line). We observe up to $3 \mathrm{~dB}$ isolation with an electrical input of 25 $\mathrm{dBm}$, and smaller values as the input decreases. The dashed lines are the fitting considering the conversion equations. In the bottom right we show the maximum isolation expected and the bandwidth of conversion for the dispersion extracted from the fitting of the measured data.

The spectral width of the dip corresponds to the bandwidth of the device and is limited by the group velocity mismatch of the even and odd modes. In our measured spectrum (Fig. 4), we see significant nonreciprocity over a bandwidth of $200 \mathrm{GHz}$. This bandwidth is much larger than the modulation frequency used. This result provides a direct experimental proof that the operating bandwidth of the device is not limited by the available modulation frequency, and thus a proof-of-principle demonstration that broadband operation of such a device is achievable. Theoretically, it has been pointed out that the bandwidth of such a device can exceed $\mathrm{THz}$ for $10 \mathrm{GHz}$ modulation frequency [24].

Our demonstration of nonreciprocity provides an important step toward integrated nonmagnetic optical isolator on a silicon chip. In addition to the bandwidth consideration as discussed above, the isolation ratio, insertion loss, and power efficiency of the device can be further improved using appropriate design and fabrication of the waveguide and electrical elements. The isolation ratio can be increased by having better impedance matching and higher input power for the electrical signal. There is no fundamental limit on the isolation ratio that one can obtain in the present scheme. For this specific device, the insertion loss is around $70 \mathrm{~dB}$ primarily due to the waveguide scattering loss, which can be significantly reduced with better fabrication processes. Modulation loss caused by diodes will ultimately limit the insertion loss. In our current design, the dopants are expected to induce loss on the order of $16 \mathrm{~dB}$ 
for a $1.0 \mathrm{~cm}$ device. This loss could be decreased greatly by using alternative $p n$-junction schemes such as recently demonstrated in [25], which also reduce the power consumption and increase the isolation attained.

Supplementary information.-We fabricate the device on a SOI platform in a completely complementary metaloxide semiconductor (CMOS)-compatible process. The polymethyl methacrylate (PMMA) photoresist masks for the dopants are written using $e$-beam lithography, followed by implantation of $\mathrm{B}^{+}$with a concentration of $1 \times$ $10^{17} \mathrm{~cm}^{-3}$ and the $\mathrm{P}^{-}$with a concentration of $1 \times$ $10^{18} \mathrm{~cm}^{-3}$. Masks for highly doped regions are written as well, followed by implantation of $1 \times 10^{20} \mathrm{~cm}^{-3}$ of $\mathrm{BF}_{2}{ }^{+}$and $\mathrm{Ar}^{-}$to form a low-resistance region for accessing the $p$ and $n$ regions, respectively, for electrical contacts. Next we write a maN-2403 photoresist mask with the waveguide pattern using $e$-beam lithography, and etch the silicon down $215 \mathrm{~nm}$, leaving a thin 35-nm slab everywhere. The dopants are then activated in an anneal furnace and a rapid thermal anneal (RTA) process, followed by cladding the waveguides with a $1-\mu \mathrm{m}$ thick $\mathrm{SiO}_{2}$ deposited with a plasma-enhanced chemical vapor deposition (PECVD) tool. We then write the mask for vias and inductors, etching through the $\mathrm{SiO}_{2}$ and sputtering $100 \mathrm{~nm}$ of $\mathrm{MoSi}_{2}$ for low-resistance contacts and $600 \mathrm{~nm}$ of $\mathrm{AlCuSi}$. Another cladding layer is deposited, 600-nm thick, and a second set of vias is etched. Finally, we write the mask for liftoff of a $1500 \mathrm{~nm}$ deposition of AlCuSi to fill up the vias and form the transmission lines. In the left side of Fig. 4, we show an image of the electrical elements of the isolator and of the optical elements of the fabricated device. The insets point out to the inductors we place in parallel to the diodes, and to the vias which contact the $p n-n p$ diodes.

For the simulation shown in Fig. 2(b), the input signal was set at a wavelength of $1550 \mathrm{~nm}$, and the permittivity modulation has a maximum $\Delta \varepsilon=0.23211$, at $10 \mathrm{THz}$ with wave number $q=541391 \mathrm{~m}^{-1}$. The total length of the waveguide is $30 \mu \mathrm{m}$. Also, simulations with modulation frequency reduced down to $100 \mathrm{GHz}$ were successfully performed, with $q=25984.8 \mathrm{~m}^{-1}, \Delta \varepsilon=0.0182$ and device length of $0.413 \mathrm{~mm}$, with mode conversion observed in only one direction. These parameters are chosen so that we can use full field simulations to highlight the essential physics, and to validate the coupled mode theory model. The experimental modulation frequency is at $10 \mathrm{GHz}$, and the theoretical model that is directly compared to the experiments is a coupled mode theory model.

This work was performed in part at the Cornell NanoScale Facility, a member of the National Nanotechnology Infrastructure Network, which is supported by the National Science Foundation. This work was supported in part by the NSF through CIAN ERC under Grant EEC-0812072. Hugo Lira thanks his sponsorship support provided by the Brazilian Defense Ministry and acknowledges insightful discussions with Professor Ehsan Afshari. The authors also acknowledge the support in part of the AFOSR-MURI program (Grant No. FA955009-1-0704).

*Corresponding author. michal.lipson@cornell.edu

[1] Z. Yu, G. Veronis, Z. Wang, and S. Fan, Phys. Rev. Lett. 100, 023902 (2008).

[2] F. D. M. Haldane and S. Raghu, Phys. Rev. Lett. 100, 013904 (2008).

[3] Z. Wang, Y. Chong, J. D. Joannopoulos, and M. Soljacic, Nature (London) 461, 772 (2009).

[4] S. Fan, R. Baets, A. Petrov, Z. Yu, J. D. Joannopoulos, W. Freude, A. Melloni, M. Popović, M. Vanwolleghem, D. Jalas, M. Eich, M. Krause, H. Renner, E. Brinkmeyer, and C. R. Doerr, Science 335, 38 (2012).

[5] J. N. Winn, S. H. Fan, J. D. Joannopoulos, and E. P. Ippen, Phys. Rev. B 59, 1551 (1999).

[6] Z. Yu and S. Fan, Nature Photon. 3, 91 (2009).

[7] K. Gallo, G. Assanto, K. R. Parameswaran, and M. M. Fejer, Appl. Phys. Lett. 79, 314 (2001).

[8] M. Soljacic, C. Luo, J. D. Joannopoulos, and S. H. Fan, Opt. Lett. 28, 637 (2003).

[9] S. K. Ibrahim, S. Bhandare, D. Sandel, H. Zhang, and R. Noe, Electron. Lett. 40, 1293 (2004).

[10] S. Manipatruni, J. T. Robinson, and M. Lipson, Phys. Rev. Lett. 102, 213903 (2009).

[11] C. R. Doerr, N. Dupuis, and L. Zhang, Opt. Lett. 36, 4293 (2011).

[12] L. Fan, J. Wang, L. T. Varghese, H. Shen, B. Niu, Y. Xuan, A. M. Weiner, and M. Qi, Science 335, 447 (2011).

[13] M. S. Kang, A. Butsch, and P. S. J. Russell, Nature Photon. 5, 549 (2011).

[14] A. Kamal, J. Clarke, and M. H. Devoret, Nature Phys. 7, 311 (2011).

[15] J. Fujita, M. Levy, J. R. M. Osgood, L. Wilkens, and H. Dotsch, Appl. Phys. Lett. 76, 2158 (2000).

[16] L. Bi, J. Hu, P. Jiang, D. H. Kim, G. F. Dionne, L. C. Kimerling, and C. A. Ross, Nature Photon. 5, 758 (2011).

[17] Y. Shoji, T. Mizumoto, H. Yokoi, I.-W. Hsieh, and J. Richard, and M. Osgood, Appl. Phys. Lett. 92, 071117 (2008).

[18] W. Migaj, J. Romero-Vivas, B. Gralak, L. Magdenko, B. Dagens, and M. Vanwolleghem, Opt. Lett. 35, 568 (2010).

[19] Z. Wang and S. Fan, Opt. Lett. 30, 1989 (2005).

[20] H. A. Haus, Waves and Fields in Optoelectronics (Prentice-Hall, Englewood Cliffs, NJ, 1984).

[21] S. E. Harris and R. W. Wallace, J. Opt. Soc. Am. 59, 744 (1969).

[22] P. Dong, S. F. Preble, J. T. Robinson, S. Manipatruni, and M. Lipson, Phys. Rev. Lett. 100, 033904 (2008).

[23] Q.F. Xu, S. Manipatruni, B. Schmidt, J. Shakya, and M. Lipson, Opt. Express 15, 430 (2007).

[24] Z. Yu and S. Fan, IEEE J. Sel. Top. Quantum Electron. 16, 459 (2010).

[25] A. Khilo, C. M. Sorace, and F. X. Kärtner, Opt. Express 19, 4485 (2011). 